\title{
Success and Decisiveness on proper symmetric games
}

\author{
Josep Freixas and Montserrat Pons*
}

November 5, 2013

\begin{abstract}
This paper provides a complete study for the possible rankings of success and decisiveness for individuals in symmetric voting systems, assuming anonymous and independent probability distributions. It is proved that for any pair of symmetric voting systems it is always possible to rank success and decisiveness in opposite order whenever the common probability of voting for "acceptance" is big enough. On the contrary, for probability values lower than one-half it is not possible to reverse the ranking of these two measures.
\end{abstract}

Keywords: Success; Decisiveness; Symmetric simple voting games; Common and opposite rankings

JEL-Codes: D72

Math. Subj. Class. (2000): 91A12, 91A80

\section{Introduction}

In this paper we consider an scenario in which the members of a set of voters have to decide on an unchangeable external proposal by means of a voting rule, each voter can only vote in favor or against it, and the outcome decision is either to accept or to reject the proposal. We assume that each member decides his vote independently of the others and even from his

*Department of Applied Mathematics III and High Engineering School (Manresa Campus), Technical University of Catalonia (Spain). Research partially funded by Grants SGR 2009-1029 of Generalitat de Catalunya and MTM 2012-34426/FEDER of "Ministerio de Economía y Competitividad".

E-mail: [josep.freixas,montserrat.pons]@upc.edu. 
own vote in previous or future occasions.

Many democratic institutions make decisions according to the results obtained in a symmetrical voting system following the principle "one man one vote". In this voting context two indices excel: one of them measures the decisiveness of each voter and the other measures his success. Both indices are important in decision-making and in human resources, and the comparisons between these two indices, either in the same voting game or in two different games, are necessary to better understand the importance of voters from these two points of view.

As usual, a voting system is modeled as a simple game $(N, \mathcal{W})$, where $N=\{1,2, \ldots, n\}$ denotes the set of voters, the cardinal $|N|=n>1$, each subset of $N$ is a coalition, and $\mathcal{W}$ is a monotonic collection of coalitions, called winning coalitions. Subsets of $N$ that are not in $\mathcal{W}$ are called losing coalitions. We assume that $\emptyset \notin \mathcal{W}$ and $N \in \mathcal{W}$.

The simple games we will deal with in this paper are the symmetric games $\mathcal{W}_{k}$ on $N$, also called $k$-out-of- $n$ games, defined for any $k(1 \leq k \leq n)$ by

$$
S \in \mathcal{W}_{k} \Leftrightarrow|S| \geq k
$$

In the symmetric game $\mathcal{W}_{k}$, the number $k$ is the level of consensus of the game, since $k$ is the minimum number of voters required to approve a proposal.

Recall that a simple game is strong if two disjoint coalitions cannot be both losing, it is proper if two disjoint coalitions cannot be both winning, and it is decisive (or a constant-sum game) if it is both, strong and proper. If a game is decisive then, for any $S \subseteq N$, exactly one of the two coalitions $S$ and $N \backslash S$ is winning, so that $|\mathcal{W}|=2^{n-1}$. It is known (see May [16]) that a $k$-out-of- $n$ game $\left(N, \mathcal{W}_{k}\right)$ is decisive if and only if $n$ is odd and $k=\frac{n+1}{2}$. In this article $n$ can be odd or even, but we assume that $k>\frac{n}{2}$, which implies that the game $\left(N, \mathcal{W}_{k}\right)$ is proper.

Before the votes are cast it is not possible to know which coalition (formed by the "yes" voters) will emerge, but we assume that an estimation $\mathfrak{p}(S)$ is made of the probability of occurrence of each vote configuration $S$ that may arise.

In this paper we assume that, for any $S \subseteq N$, the probability $\mathfrak{p}(S)$ is given by

$$
\mathfrak{p}(S)=p^{s}(1-p)^{n-s}
$$

where $p$ is a fixed value in $[0,1]$ and $s$ stands for $|S|$. This is equivalent to assuming that each voter, independently of the others, votes "yes" with probability $p$ and votes "no" with probability $1-p$. Laruelle and Valenciano ([15]) refer to this distribution of probability as being anonymous and independent.

The idea of decisiveness has been widely used as a basis for the most well-known power indices such as the Shapley-Shubik index ([22], [23], [7]) or the four proportional indices: 
Penrose ([20]), Banzhaf ([1]), Coleman to prevent action and Coleman to initiate action ([6]), and many authors have referred to this aspect when analyzing power indices (see, for example, [11], [17], [24] or [25]). But only a few authors before A. Laruelle and F. Valenciano had referred to the idea of success (the likelihood of obtaining the result that one votes for) as being also a relevant point to take into account when measuring voting power (see [2], [3], [4], [12], [21], [19]). The sound and lucid analysis made by Laruelle and Valenciano about these concepts in [14], [13] and [15] lead us to work on the cases described in this paper. Being more specific, they proved in [13] that success and decisiveness are in general not only conceptually different but also analytically independent, and they showed in [15] (Example 3.1) that two symmetric games can be differently ranked from these two points of view.

The goal of our work is to compare the success and the decisiveness of a voter in symmetric games, with anonymous and independent probability distribution, for different levels of consensus. We also compare, by using a convenient scale, the values of the two indices for a voter in a fixed game of the same kind. In section 2 the definitions of the success (ex ante) index $\Omega_{i}$, and the decisiveness (ex ante) index $\Phi_{i}$ of a voter $i$ are recalled, and their expressions for the cases under study are shown. In section 3 we compare the decisiveness index of a voter for different levels of consensus $k$, and an analogous study is done in section 4 for the success index. Section 5 is devoted to compare $\Omega$ and $\frac{1}{2}+\frac{1}{2} \Phi$, for a fixed $k$ and for all possible values of $p \in[0,1]$, since it is known that these two values coincide when $p=\frac{1}{2}$. Finally, section 6 summarizes the work and points out the main conclusions.

\section{Success and decisiveness ex ante}

In this section we recall the definitions of success and decisiveness ex ante given in [13] or [14] and evaluate them in our particular cases.

\subsection{Estimating a voter's ex ante success}

After a decision is made, a voter is considered to have been successful if his vote happens to be in accordance with the result, that is to say, if either he voted in favor of the proposal and it has been accepted or he voted against the proposal and it has been rejected. Before the votes are cast, the voter $i$ 's (ex ante) success is the probability that $i$ is successful:

\section{Definition 2.1}

$$
\Omega_{i}(\mathcal{W}, \mathfrak{p})=\sum_{\substack{S \subseteq N \\ i \in S \in \mathcal{W}}} \mathfrak{p}(S)+\sum_{\substack{S \subseteq N \\ i \notin S \notin \mathcal{W}}} \mathfrak{p}(S)
$$


Assuming that $\left(N, \mathcal{W}_{k}\right)$ is a $k$-out-of- $n$ game, $p$ is a fixed value in $[0,1]$, and $\mathfrak{p}(S)=$ $p^{s}(1-p)^{n-s}$ for any $S \subseteq N$, then we write $\Omega\left(\mathcal{W}_{k}, p\right)$ instead of $\Omega_{i}\left(\mathcal{W}_{k}, \mathfrak{p}\right)$ since its value is the same for any voter $i$ and $\mathfrak{p}$ depends only of $p$. In this case it is:

$$
\Omega\left(\mathcal{W}_{k}, p\right)=\sum_{s=k}^{n}\left[\left(\begin{array}{c}
n-1 \\
s-1
\end{array}\right) p^{s}(1-p)^{n-s}\right]+\sum_{s=0}^{k-1}\left[\left(\begin{array}{c}
n-1 \\
s
\end{array}\right) p^{s}(1-p)^{n-s}\right] .
$$

If $p=\frac{1}{2}$ this index $\Omega$ is precisely the Rae index, introduced in [21] for symmetric games and extended to any simple game in [8]. Notice also that $\Omega\left(\mathcal{W}_{k}, 0\right)=\Omega\left(\mathcal{W}_{k}, 1\right)=1$.

Let us see that the expression of $\Omega\left(\mathcal{W}_{k}, p\right)$ can be written in another way that will be used further on in this paper.

Using that

$$
\begin{aligned}
1 & =(p+1-p)^{n-1}=\sum_{s=0}^{n-1}\left[\left(\begin{array}{c}
n-1 \\
s
\end{array}\right) p^{s}(1-p)^{n-s-1}\right] \\
& =\sum_{s=0}^{k-2}\left[\left(\begin{array}{c}
n-1 \\
s
\end{array}\right) p^{s}(1-p)^{n-s-1}\right]+\sum_{s=k-1}^{n-1}\left[\left(\begin{array}{c}
n-1 \\
s
\end{array}\right) p^{s}(1-p)^{n-s-1}\right]
\end{aligned}
$$

we can write:

$$
\begin{aligned}
\Omega\left(\mathcal{W}_{k}, p\right) & =\sum_{s=k-1}^{n-1}\left[\left(\begin{array}{c}
n-1 \\
s
\end{array}\right) p^{s+1}(1-p)^{n-s-1}\right]+\sum_{s=0}^{k-1}\left[\left(\begin{array}{c}
n-1 \\
s
\end{array}\right) p^{s}(1-p)^{n-s}\right] \\
& =p-\sum_{s=0}^{k-2}\left[\left(\begin{array}{c}
n-1 \\
s
\end{array}\right) p^{s+1}(1-p)^{n-s-1}\right]+\sum_{s=0}^{k-1}\left[\left(\begin{array}{c}
n-1 \\
s
\end{array}\right) p^{s}(1-p)^{n-s}\right] \\
& =p+\left(\begin{array}{c}
n-1 \\
k-1
\end{array}\right) p^{k-1}(1-p)^{n-k+1}+(1-2 p) \sum_{s=0}^{k-2}\left[\left(\begin{array}{c}
n-1 \\
s
\end{array}\right) p^{s}(1-p)^{n-s-1}\right] .
\end{aligned}
$$

\subsection{Estimating a voter's ex ante decisiveness}

After a decision is made, a voter is considered to have been decisive if he was successful and his vote was critical to that success. Before the votes are cast, the voter $i$ 's (ex ante) decisiveness is the probability that $i$ is decisive:

\section{Definition 2.2}

$$
\Phi_{i}(\mathcal{W}, \mathfrak{p})=\sum_{\substack{S \subseteq N \\ i \in S \in \mathcal{W} \\ S \backslash\{i\} \notin \mathcal{W}}} \mathfrak{p}(S)+\sum_{\substack{S \subseteq N \\ i \notin S \notin \mathcal{W} \\ S \cup\{i\} \in \mathcal{W}}} \mathfrak{p}(S)
$$


Assuming that $\left(N, \mathcal{W}_{k}\right)$ is a $k$-out-of- $n$ game, $p$ is a fixed value in $[0,1]$, and $\mathfrak{p}(S)=$ $p^{s}(1-p)^{n-s}$ for any $S \subseteq N$, then we write $\Phi\left(\mathcal{W}_{k}, p\right)$ instead of $\Phi_{i}\left(\mathcal{W}_{k}, \mathfrak{p}\right)$, analogously as what we did for $\Omega$ in the former subsection. Now we have:

$$
\begin{gathered}
\Phi\left(\mathcal{W}_{k}, p\right)=\left(\begin{array}{c}
n-1 \\
k-1
\end{array}\right) p^{k}(1-p)^{n-k}+\left(\begin{array}{c}
n-1 \\
k-1
\end{array}\right) p^{k-1}(1-p)^{n-k+1} \\
\Phi\left(\mathcal{W}_{k}, p\right)=\left(\begin{array}{c}
n-1 \\
k-1
\end{array}\right) p^{k-1}(1-p)^{n-k} .
\end{gathered}
$$

If $p=\frac{1}{2}$ this index $\Phi$ is precisely the Banzhaf index ([1], [8], [18]). Notice also that $\Phi\left(\mathcal{W}_{k}, 0\right)=0, \Phi\left(\mathcal{W}_{k}, 1\right)=0$ if $k<n$ and $\Phi(\mathcal{W}, 1)=1$ if $k=n$.

\section{Decisiveness of a voter in different games}

The purpose of this section is to analyze the changes in the ex ante decisiveness of a voter when the level of consensus changes, that is to say, our goal here is to compare the decisiveness of a voter for different values of $k$, in $k$-out-of- $n$ games with anonymous and independent probability distribution.

We consider two different proper symmetric games on the same set of voters: $\left(N, \mathcal{W}_{k}\right)$ and $\left(N, \mathcal{W}_{r}\right)$ with $\frac{n}{2}<k<r \leq n$. Let us denote by $\Phi^{k}(p)$ and $\Phi^{r}(p)$ the decisiveness indices corresponding to an arbitrary voter in $\left(N, \mathcal{W}_{k}\right)$ and $\left(N, \mathcal{W}_{r}\right)$, respectively. We are going to study the difference $\Phi^{k}(p)-\Phi^{r}(p)$.

$$
\begin{aligned}
\Phi^{k}(p)-\Phi^{r}(p) & =\left(\begin{array}{c}
n-1 \\
k-1
\end{array}\right) p^{k-1}(1-p)^{n-k}-\left(\begin{array}{c}
n-1 \\
r-1
\end{array}\right) p^{r-1}(1-p)^{n-r} \\
& =p^{k-1}(1-p)^{n-r}\left[\left(\begin{array}{c}
n-1 \\
k-1
\end{array}\right)(1-p)^{r-k}-\left(\begin{array}{c}
n-1 \\
r-1
\end{array}\right) p^{r-k}\right] .
\end{aligned}
$$

For any $k\left(\frac{n}{2}<k<r<n\right)$ the equation $\Phi^{k}(p)-\Phi^{r}(p)=0$ has three different roots: $p=0$, $p=1$ and $p=p_{k, r}$ defined by:

$$
p_{k, r}=\left[\left(\frac{(k-1) !(n-k) !}{(r-1) !(n-r) !}\right)^{\frac{1}{r-k}}+1\right]^{-1}
$$

For $r=n$ the equation $\Phi^{k}(p)-\Phi^{r}(p)=0$ has only the roots $p=0$ and $p=p_{k, r}$, because in this case $\Phi^{k}(1)-\Phi^{2 n-1}(1)=-1$.

In the following theorem some properties of the roots $p_{k, r}$ are established.

\section{Theorem 3.1}

Let $k, r$ be such that $\frac{n}{2}<k<r \leq n$, and $p_{k, r}$ be the number defined in (4). 
a) $p_{k, r} \in\left(\frac{1}{2}, 1\right)$.

b) $p_{k, k+1}=\frac{k}{n}$.

c) If $r<n$ then $p_{k, r}<p_{k, r+1}$.

d) If $k>\frac{n}{2}+1$ then $p_{k-1, r}<p_{k, r}$.

e) $p_{k, r} \leq \frac{n-1}{n}$.

\section{Proof:}

To see that $p_{k, r} \in\left(\frac{1}{2}, 1\right)$ first notice that

$$
\frac{(k-1) !(n-k) !}{(r-1) !(n-r) !}=\prod_{j=1}^{r-k} \frac{n-k-j+1}{r-j} .
$$

It is clear that (5) is strictly positive, and, since $k+r>n$, it is $\frac{n-k-j+1}{r-j}<1$ for any $j(1 \leq j \leq r-k)$, so that (5) is strictly lower than 1. Then, from (4) we deduce that $\frac{1}{2}<p_{k, r}<1$.

Part b) is obtained directly from the expression (4) with $r=k+1$.

To prove part c) we need to assume that $r<n$ and see that

$$
\left(\frac{(k-1) !(n-k) !}{(r-1) !(n-r) !}\right)^{\frac{1}{r-k}}>\left(\frac{(k-1) !(n-k) !}{r !(n-r-1) !}\right)^{\frac{1}{r+1-k}} .
$$

This inequality is equivalent to

$$
\left(\frac{(k-1) !(n-k) !}{(r-1) !(n-r) !}\right)^{\frac{1}{r-k}+1}>\frac{(k-1) !(n-k) !}{r !(n-r-1) !}
$$

and also equivalent to

$$
\left(\frac{(k-1) !(n-k) !}{(r-1) !(n-r) !}\right)^{\frac{1}{r-k}}>\frac{n-r}{r}
$$

But this last inequality is true because $\frac{n-k-j+1}{r-j}>\frac{n-r}{r}$ for any $j(1 \leq j \leq r-k)$, and using (5) we have

$$
\frac{(k-1) !(n-k) !}{(r-1) !(n-r) !}>\left(\frac{n-r}{r}\right)^{r-k} .
$$

In a similar way, to prove part d) we assume that $k>\frac{n}{2}+1$ and see that

$$
\left(\frac{(k-1) !(n-k) !}{(r-1) !(n-r) !}\right)^{\frac{1}{r-k}}<\left(\frac{(k-2) !(n-k+1) !}{(r-1) !(n-r) !}\right)^{\frac{1}{r+1-k}}
$$

This inequality is equivalent to

$$
\left(\frac{(k-1) !(n-k) !}{(r-1) !(n-r) !}\right)^{\frac{1}{r-k}+1}<\frac{(k-2) !(n-k+1) !}{(r-1) !(n-r) !}
$$


and also equivalent to

$$
\left(\frac{(k-1) !(n-k) !}{(r-1) !(n-r) !}\right)^{\frac{1}{r-k}}<\frac{n-k+1}{k-1} .
$$

But this is true because $\frac{n-k-j+1}{r-j}<\frac{n-k+1}{k-1}$ for any $j(1 \leq j \leq r-k)$, and using (5) it is

$$
\frac{(k-1) !(n-k) !}{(r-1) !(n-r) !}<\left(\frac{n-k+1}{k-1}\right)^{r-k} .
$$

As a consequence of parts c) and d) it is always $p_{k, r} \leq p_{n-1, n}$. From part b) it is $p_{n-1, n}=\frac{n-1}{n}$ so that

$$
p_{k, r} \leq \frac{n-1}{n} \text {. }
$$

As a consequence of Theorem 3.1 we observe that for any $k, r$ with $\frac{n}{2}<k<r \leq n$, the difference $\Phi^{k}(p)-\Phi^{r}(p)$ has different sign depending on $p$ being lower than $p_{k, r}$ or greater than it. Theorem 3.1-a) and Theorem 3.1-e) give bounds for the change of sign of the difference $\Phi^{k}(p)-\Phi^{r}(p)$, so that this change always occurs in the interval $\left(\frac{1}{2}, \frac{n-1}{n}\right]$. Theorem 3.1-c) and Theorem 3.1-d) tell us that if the consensus level is increased in only one of the two games then the sign change occurs at a higher value. Theorem 3.1-b) gives the value of sign change for two consecutive levels of consensus. And, finally, if we combine parts $b$ ), c) and $d$ ) of Theorem 3.1 we can observe that:

- The minimum value for $p_{k, r}$ is $\frac{\left\lfloor\frac{n}{2}\right\rfloor+1}{n}$, and it is attained when $k$ gives the absolute majority rule and $r=k+1$.

- The maximum value for $p_{k, r}$ is $\frac{n-1}{n}$, and it is attained for $r=n$ (unanimity rule) and $k=r-1$.

The sign of the difference $\Phi^{k}(p)-\Phi^{r}(p)$ is established in the following proposition.

\section{Proposition 3.2}

Let $k, r$ be such that $\frac{n}{2}<k<r \leq n$, and $p_{k, r}$ be the number defined in (4). Then, $\Phi^{k}(p)>\Phi^{r}(p)$ for any $p \in\left(0, p_{k, r}\right)$ and $\Phi^{k}(p)<\Phi^{r}(p)$ for any $p \in\left(p_{k, r}, 1\right)$.

\section{Proof:}

To prove this we are going to verify that $\Phi^{k}\left(\frac{1}{2}\right)-\Phi^{r}\left(\frac{1}{2}\right)>0$.

$$
\begin{aligned}
\Phi^{k}\left(\frac{1}{2}\right)-\Phi^{r}\left(\frac{1}{2}\right) & =\left(\frac{1}{2}\right)^{n-1}\left[\left(\begin{array}{l}
n-1 \\
k-1
\end{array}\right)-\left(\begin{array}{l}
n-1 \\
r-1
\end{array}\right)\right] \\
& =\left(\frac{1}{2}\right)^{n-1} \frac{(n-1) !}{(r-1) !(n-r) !}\left[\prod_{j=1}^{r-k}(r-j)-\prod_{j=1}^{r-k}(n-k-j+1)\right],
\end{aligned}
$$


and this expression is strictly positive because $k+r>n+1$ and so $n-k-j+1<r-j$ for any $j$. Thus, $\Phi^{k}(p)-\Phi^{r}(p)>0$ for any $p \in\left(0, p_{k, r}\right)$ and $\Phi^{k}(p)-\Phi^{r}(p)<0$ for any $p \in\left(p_{k, r}, 1\right)$.

By combining Theorem 3.1 and Proposition 3.2 we can establish that, in any case, there are values of $p$ for which $\Phi^{k}(p)>\Phi^{r}(p)$, and other values of $p$ for which $\Phi^{k}(p)<\Phi^{r}(p)$.

\section{Corollary 3.3}

Let $k, r$ be such that $\frac{n}{2}<k<r \leq n$. Then, $\Phi^{k}(p)>\Phi^{r}(p)$ for any $p \in\left(0, \frac{1}{2}\right)$ and $\Phi^{k}(p)<\Phi^{r}(p)$ for any $p \in\left(\frac{n-1}{n}, 1\right)$.

Figure 1 shows the graph of $\Phi^{k}(p)-\Phi^{r}(p)$ for $n=7$ and all possible $k, r$ with $\frac{n}{2}<k<r \leq n$.

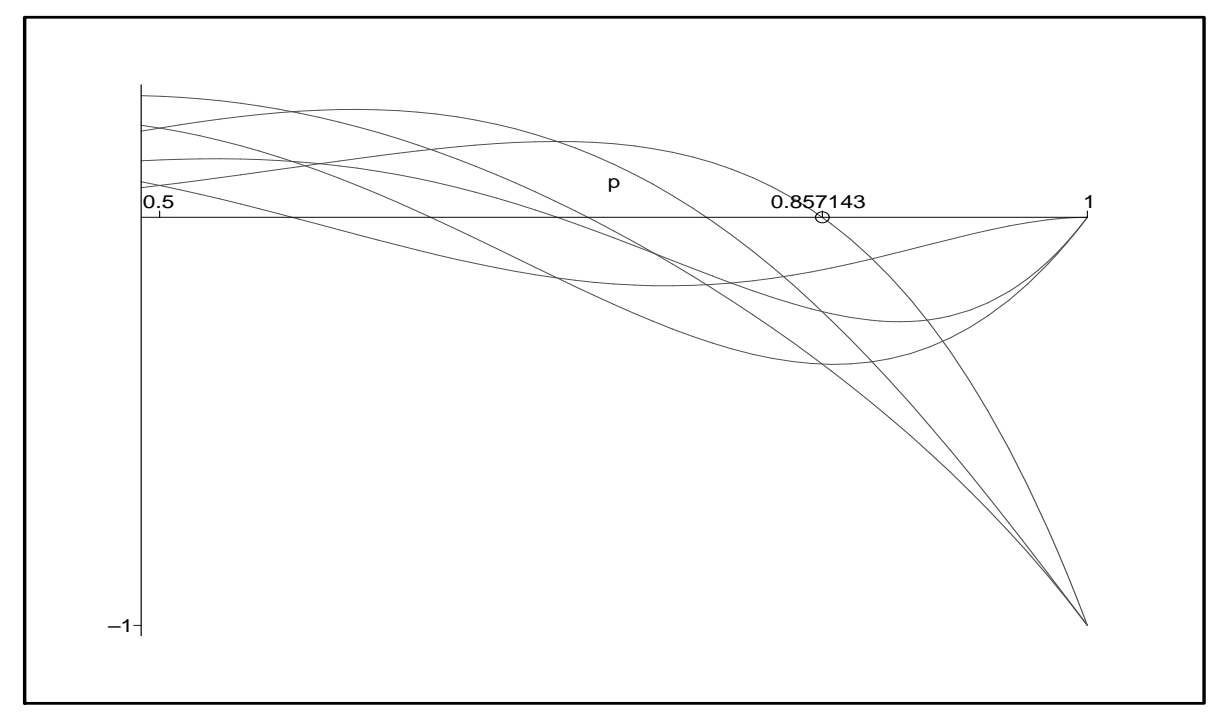

Figure 1: $\Phi^{k}(p)-\Phi^{r}(p)$ for $n=7$ and all possible $k, r$ with $\frac{n}{2}<k<r \leq n$.

Independently of the levels of consensus $k$ and $r$ of the symmetric games $\mathcal{W}_{k}$ and $\mathcal{W}_{r}$, we have seen that for $p<\frac{1}{2}$ a lower level of consensus in the game implies a greater decisiveness for voters, whereas if $p$ is close to 1 (more precisely, if $p$ takes any value greater than $1-\frac{1}{n}$ ) then this relationship is reversed. Between $p=\frac{1}{2}$ and $p=1-\frac{1}{n}$ this relationship depends on the levels of consensus $k$ and $r$, and it is detailed in Proposition 3.2. The only choices of $k$ and $r$ that lead to the upper bound $1-\frac{1}{n}$ for $p_{k, r}$ are $k=n-1$ and $r=n$ (unanimity rule), but even in this situation the change of sign of the difference $\Phi^{k}(p)-\Phi^{r}(p)$ is guaranteed. This fact becomes crucial, since, as we are going to see in the next section, the success index behaves more regularly than decisiveness index. 


\section{Success of a voter in different games}

As was done for the decisiveness in Section 3, the purpose of this section is to analyze the changes in the ex ante success of a voter when the level of consensus changes, that is to say, our goal here is to compare the success of a voter for different values of $k$, in $k$-out-of- $n$ games with anonymous and independent probability distribution.

We consider two different proper symmetric games on the same set of voters: $\left(N, \mathcal{W}_{k}\right)$ and $\left(N, \mathcal{W}_{r}\right)$ with $\frac{n}{2}<k<r \leq n$. Let us denote by $\Omega^{k}(p)$ and $\Omega^{r}(p)$ the success indices corresponding to an arbitrary voter in the first and the second mentioned games, respectively. We are going to study the difference $\Omega^{k}(p)-\Omega^{r}(p)$.

\section{Theorem 4.1}

$\Omega^{k}(p)-\Omega^{r}(p) \geq 0$ for any $p \in[0,1]$, and the inequality is strict if $p \in(0,1)$.

\section{Proof:}

Using (1) we can write:

$$
\begin{aligned}
\Omega^{k}(p)-\Omega^{r}(p) & =\sum_{s=k}^{r-1}\left(\begin{array}{c}
n-1 \\
s-1
\end{array}\right) p^{s}(1-p)^{n-s}-\sum_{s=k}^{r-1}\left(\begin{array}{c}
n-1 \\
s
\end{array}\right) p^{s}(1-p)^{n-s} \\
& =\sum_{s=k}^{r-1} p^{s}(1-p)^{n-s}\left[\left(\begin{array}{c}
n-1 \\
s-1
\end{array}\right)-\left(\begin{array}{c}
n-1 \\
s
\end{array}\right)\right] \\
& =\sum_{s=k}^{r-1} p^{s}(1-p)^{n-s} \frac{(n-1) !}{s !(n-s) !}(2 s-n) \geq 0
\end{aligned}
$$

because $2 s-n>0$ for any $s \geq k$.

Figure 2 shows the graph of $\Omega^{k}(p)-\Omega^{r}(p)$ for $n=7$ and all possible $k, r$ with $\frac{n}{2}<k<r \leq n$.

Theorem 4.1 tells us that, no matter which are the levels of consensus to be compared, the lower the level of consensus demanded the higher the index of success of the voters. Putting together the results obtained in the previous and in the present section we may compare Proposition 3.2 and Theorem 4.1 to deduce that the indices of decisiveness and success rank voters in a different way depending on the values of $p$.

\section{Corollary 4.2}

Let $k, r$ be such that $\frac{n}{2}<k<r \leq n$, and $p_{k, r}$ be the number defined in (4). Then,

a) If $p \in\left(0, p_{k, r}\right)$ then $\Omega^{k}(p)>\Omega^{r}(p)$ and $\Phi^{k}(p)>\Phi^{r}(p)$.

b) If $p \in\left(p_{k, r}, 1\right)$ then $\Omega^{k}(p)>\Omega^{r}(p)$ and $\Phi^{k}(p)<\Phi^{r}(p)$. 


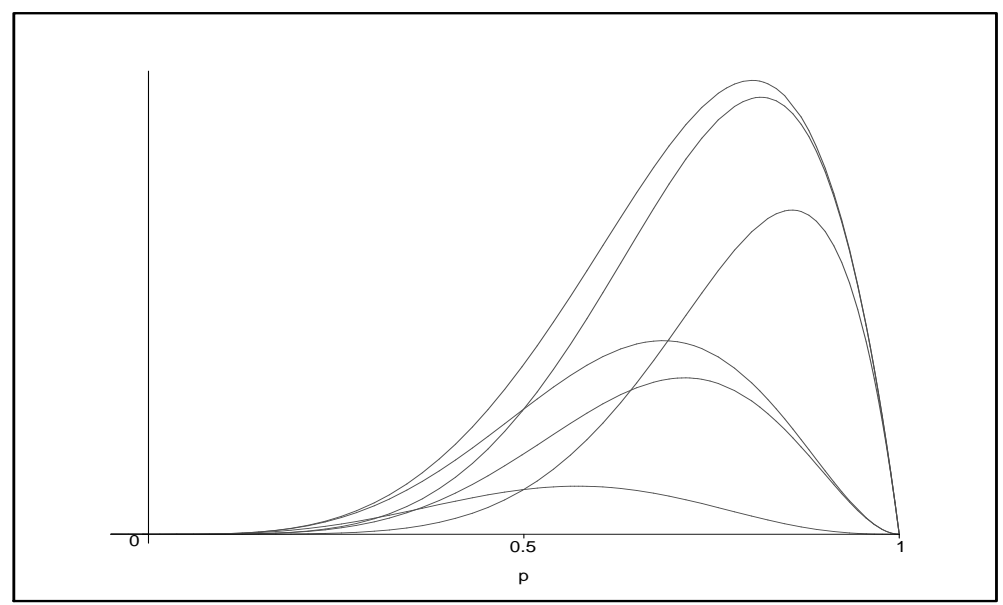

Figure 2: $\Omega^{k}(p)-\Omega^{r}(p)$ for $n=7$ and all possible $k, r$ with $\frac{n}{2}<k<r \leq n$.

Finally, by using Corollary 3.3 and Theorem 4.1, we can establish that, independently of the particular values of $k$ and $r$, for values of $p$ smaller than $\frac{1}{2}$ success and decisiveness indices rank voters in the same way, and for values of $p$ near one (more precisely, if $\frac{n-1}{n}<p<1$ ) then these two indices rank voters in an opposite way.

Notice that, while decisiveness has a quite irregular behavior for voters, success behaves always in the same way: the higher the level of consensus required the lower the success of voters. Consequently, the different rankings between success and decisiveness depend on the behavior of decisiveness. In fact, if the value of $p$ is large enough then the opposite ranking between success and decisiveness is guaranteed, independently of the values of $k, r$ and $n$.

\section{Comparing success and decisiveness in the same game}

It is obvious that $\Phi_{i}(\mathcal{W}, \mathfrak{p}) \leq \Omega_{i}(\mathcal{W}, \mathfrak{p})$ for any game $(N, \mathcal{W})$, any $i \in N$, and any vote configuration $\mathfrak{p}$. In the particular case of $\mathfrak{p}(S)=\frac{1}{2^{n}}$ for any $S \subseteq N$ it happens that $\Omega_{i}(\mathcal{W}, \mathfrak{p})=$ $\frac{1}{2}+\frac{1}{2} \Phi_{i}(\mathcal{W}, \mathfrak{p})$, as was anticipated by Penrose in [20] and proved by Dubey and Shapley in [8]. However, in general, $\Omega_{i}(\mathcal{W}, \mathfrak{p}) \neq \frac{1}{2}+\frac{1}{2} \Phi_{i}(\mathcal{W}, \mathfrak{p})$, and the equality holds for all $\mathcal{W}$ if and only if all vote configurations are equally probable (see [13]). We are going to compare these two magnitudes for different anonymous and independent probability distributions. The study in this section is nothing else than a comparison between success and decisiveness by using a convenient scale, and it complements the ordinal comparisons we have established in the two precedent sections.

Our goal in this section is to compare $\Omega\left(\mathcal{W}_{k}, p\right)$ and $\frac{1}{2}+\frac{1}{2} \Phi\left(\mathcal{W}_{k}, p\right)$, for a fixed $k$ (with $\frac{n}{2}<k \leq n$ to ensure that the game is proper) and to see whether there are other values of $p$, apart from $p=\frac{1}{2}$, for which they are coincident. 
Theorem 5.1 Let $D_{k}(p)=\Omega\left(\mathcal{W}_{k}, p\right)-\frac{1}{2}-\frac{1}{2} \Phi\left(\mathcal{W}_{k}, p\right)$. Then:

a) There are two roots of $D_{k}(p)=0$ in $[0,1]$. One of them is $\frac{1}{2}$, and the other, $p_{k}$, is in $\left[\frac{1}{2}, 1\right]$.

b) $p_{k}=\frac{1}{2}$ if and only if $n$ is odd and $k=\frac{n+1}{2}$.

In this case $\frac{1}{2}$ is a double root of $D_{k}(p)=0$.

c) $p_{k}<p_{k+1}$ for any $k$ with $\frac{n}{2}<k \leq n$.

d) $p_{n}=1$.

Proof:

Using (2) and (3) we can write:

$$
\begin{aligned}
D_{k}(p) & =\Omega\left(\mathcal{W}_{k}, p\right)-\frac{1}{2}-\frac{1}{2} \Phi\left(\mathcal{W}_{k}, p\right) \\
& =p-\frac{1}{2}+\left(\begin{array}{c}
n-1 \\
k-1
\end{array}\right) p^{k-1}(1-p)^{n-k}\left(\frac{1}{2}-p\right)+(1-2 p) \sum_{s=0}^{k-2}\left[\left(\begin{array}{c}
n-1 \\
s
\end{array}\right) p^{s}(1-p)^{n-s-1}\right] \\
& =\left(\frac{1}{2}-p\right)\left[-1+\left(\begin{array}{c}
n-1 \\
k-1
\end{array}\right) p^{k-1}(1-p)^{n-k}+2 \sum_{s=0}^{k-2}\left[\left(\begin{array}{c}
n-1 \\
s
\end{array}\right) p^{s}(1-p)^{n-s-1}\right]\right] .
\end{aligned}
$$

Thus, it is clear that $p=\frac{1}{2}$ is a root of $D_{k}(p)=0$ independently of the values of $k$ and $n$. Let us prove that there is another root of $D_{k}(p)=0$ in $\left[\frac{1}{2}, 1\right]$. To this end, we will prove that the equation $M_{k}(p)=0$ has a unique root $p_{k}$ in $\left[\frac{1}{2}, 1\right]$, where $M_{k}(p)$ is given by

$$
M_{k}(p)=-1+\left(\begin{array}{c}
n-1 \\
k-1
\end{array}\right) p^{k-1}(1-p)^{n-k}+2 \sum_{s=0}^{k-2}\left[\left(\begin{array}{c}
n-1 \\
s
\end{array}\right) p^{s}(1-p)^{n-s-1}\right] .
$$

\section{Existence:}

It is easy to check that for $k=n$ it is $M_{n}(1)=0$, which proves that a root of $M_{n}(p)=0$ is $p_{n}=1$.

For the remaining values of $k\left(\frac{n}{2}<k<n\right)$ it is $M_{k}(1)=-1$, and we will see that $M_{k}\left(\frac{1}{2}\right) \geq 0$. From Bolzano's theorem, this will prove that there is at least one root of $M_{k}(p)=0$ in $\left[\frac{1}{2}, 1\right]$.

$$
M_{k}\left(\frac{1}{2}\right)=-1+\left(\frac{1}{2}\right)^{n-1}\left[\left(\begin{array}{l}
n-1 \\
k-1
\end{array}\right)+2 \sum_{s=0}^{k-2}\left(\begin{array}{c}
n-1 \\
s
\end{array}\right)\right] .
$$

But, taking into account that

$$
2^{n-1}=\sum_{s=0}^{n-1}\left(\begin{array}{c}
n-1 \\
s
\end{array}\right) \leq\left(\begin{array}{c}
n-1 \\
k-1
\end{array}\right)+2 \sum_{s=0}^{k-2}\left(\begin{array}{c}
n-1 \\
s
\end{array}\right)
$$


it is

$$
\left(\frac{1}{2}\right)^{n-1}\left[\left(\begin{array}{l}
n-1 \\
k-1
\end{array}\right)+2 \sum_{s=0}^{k-2}\left(\begin{array}{c}
n-1 \\
s
\end{array}\right)\right] \geq 1
$$

and this proves that $M_{k}\left(\frac{1}{2}\right) \geq 0$. Notice that the equality holds if and only if $n$ is odd and $k=\frac{n+1}{2}$, i.e., when the game is decisive. In this case, a root of $M_{\frac{n+1}{2}}(p)=0$ is $p_{\frac{n+1}{2}}=\frac{1}{2}$. Thus, if $\frac{n+1}{2}<k<n$ then $M_{k}\left(\frac{1}{2}\right)>0$ and $M_{k}(1)<0$, so that there exist at least a value $p_{k} \in\left(\frac{1}{2}, 1\right)$ such that $M_{k}\left(p_{k}\right)=0$.

\section{Uniqueness:}

We will see now that $\frac{d M_{k}}{d p}<0$ for any $p \in(0,1)$. This will prove that $p_{k}$ is the only root of $M_{k}(p)=0$ in $(0,1)$. By differentiating (6) with respect to $p$ we get:

$$
\begin{aligned}
\frac{d M_{k}}{d p}= & \left(\begin{array}{c}
n-1 \\
k-1
\end{array}\right)(k-1) p^{k-2}(1-p)^{n-k}-\left(\begin{array}{c}
n-1 \\
k-1
\end{array}\right)(n-k) p^{k-1}(1-p)^{n-k-1} \\
& +2 \sum_{s=1}^{k-2}\left[\left(\begin{array}{c}
n-1 \\
s
\end{array}\right) s p^{s-1}(1-p)^{n-s-1}\right]-2 \sum_{s=0}^{k-2}\left[\left(\begin{array}{c}
n-1 \\
s
\end{array}\right)(n-s-1) p^{s}(1-p)^{n-s-2}\right] .
\end{aligned}
$$

Now, if we take $s=t+1$ in the first summation operator with index $s$, take $s=t$ in the second one, and add up these terms separately we get, for $0 \leq t \leq k-3$ :

$$
\begin{aligned}
& 2\left(\begin{array}{c}
n-1 \\
t+1
\end{array}\right)(t+1) p^{t}(1-p)^{n-t-2}-2\left(\begin{array}{c}
n-1 \\
t
\end{array}\right)(n-t-1) p^{t}(1-p)^{n-t-2} \\
= & 2 p^{t}(1-p)^{n-t-2}(n-1) !\left[\frac{t+1}{(t+1) !(n-t-2) !}-\frac{n-t-1}{t !(n-t-1) !}\right]=0 .
\end{aligned}
$$

Thus,

$$
\begin{aligned}
\frac{d M_{k}}{d p}= & \left(\begin{array}{l}
n-1 \\
k-1
\end{array}\right)(k-1) p^{k-2}(1-p)^{n-k}-\left(\begin{array}{l}
n-1 \\
k-1
\end{array}\right)(n-k) p^{k-1}(1-p)^{n-k-1}- \\
& -2\left(\begin{array}{l}
n-1 \\
k-2
\end{array}\right)(n-k+1) p^{k-2}(1-p)^{n-k} \\
= & -\left(\begin{array}{l}
n-1 \\
k-1
\end{array}\right) p^{k-2}(1-p)^{n-k-1}[(1-p)(k-1)+p(n-k)] .
\end{aligned}
$$

This proves that $\frac{d M_{k}}{d p}<0$ for any $p \in(0,1)$, because $k \geq 1, n \geq k$, with at least one of these inequalities being strict (we are assuming that $n>1$ ), ensures that $(1-p)(k-1)+p(n-k)>0$. Thus, the existence of a unique value $p_{k} \in\left[\frac{1}{2}, 1\right]$ such that $M_{k}\left(p_{k}\right)=0$ is ensured. It is clear now that $p_{n}=1$ (part $d$ ) of the theorem), and that $p_{k}=\frac{1}{2}$ if and only if $n$ is odd and $k=\frac{n+1}{2}$ (part $\left.b\right)$ of the theorem). In all other cases $p_{k} \in\left(\frac{1}{2}, 1\right)$.

Finally, to prove part $c$ ) we will see that $M_{k+1}\left(p_{k}\right)>0$. From (6) it is:

$$
M_{k+1}\left(p_{k}\right)=-1+\left(\begin{array}{c}
n-1 \\
k
\end{array}\right) p_{k}^{k}\left(1-p_{k}\right)^{n-k-1}+2 \sum_{s=0}^{k-1}\left[\left(\begin{array}{c}
n-1 \\
s
\end{array}\right) p_{k}^{s}\left(1-p_{k}\right)^{n-s-1}\right] .
$$


But

$$
M_{k}\left(p_{k}\right)=-1+\left(\begin{array}{l}
n-1 \\
k-1
\end{array}\right) p_{k}^{k-1}\left(1-p_{k}\right)^{n-k}+2 \sum_{s=0}^{k-2}\left[\left(\begin{array}{c}
n-1 \\
s
\end{array}\right) p_{k}^{s}\left(1-p_{k}\right)^{n-s-1}\right]=0
$$

so that we can write

$$
M_{k+1}\left(p_{k}\right)=\left(\begin{array}{c}
n-1 \\
k
\end{array}\right) p_{k}^{k}\left(1-p_{k}\right)^{n-k-1}+\left(\begin{array}{c}
n-1 \\
k-1
\end{array}\right) p_{k}^{k-1}\left(1-p_{k}\right)^{n-k}>0 .
$$

In Figures 3, 4, 5 and 6 we can visualize different results of Theorem 5.1.

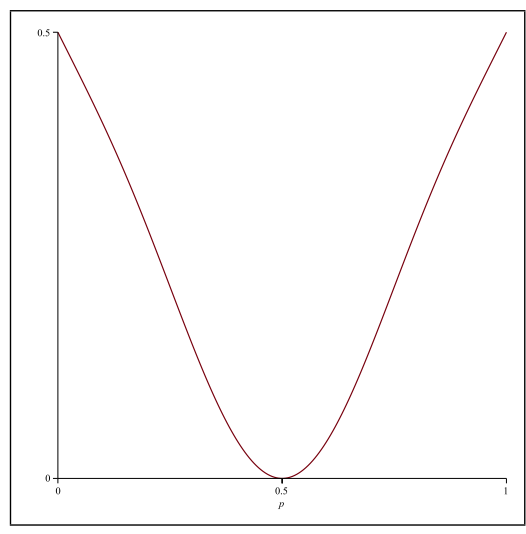

Figure 3: $D_{k}(p)$ for $n=9$ and $k=5$.

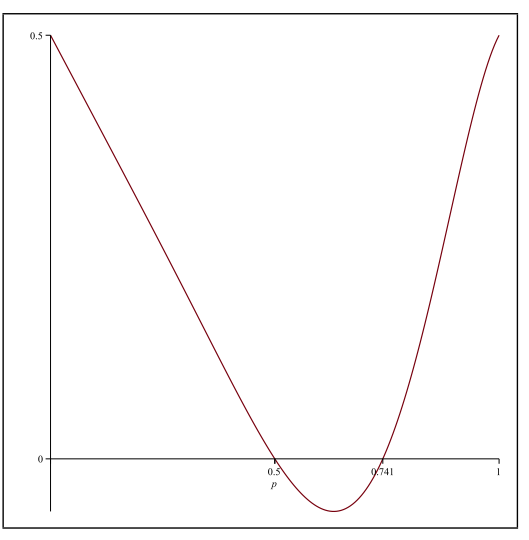

Figure 4: $D_{k}(p)$ for $n=9$ and $k=7$.

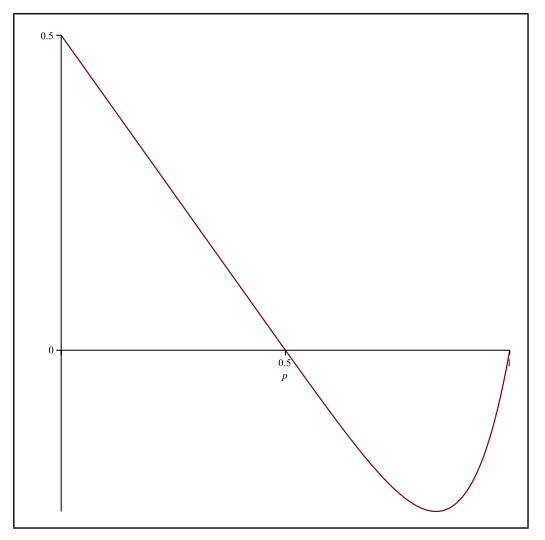

Figure 5: $D_{k}(p)$ for $n=9$ and $k=9$.

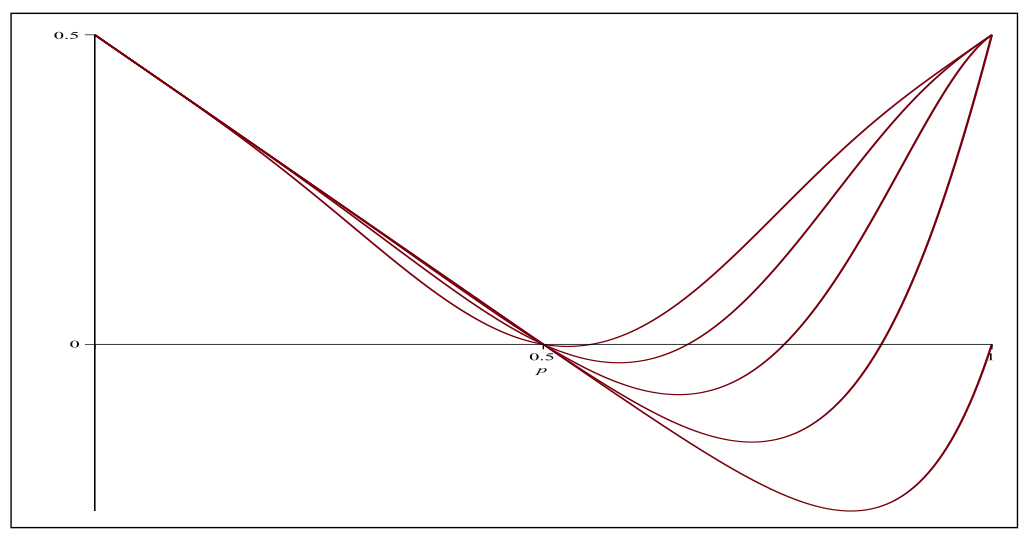

Figure 6: $D_{k}(p)$ for $n=10$ and $k=6,7,8,9,10$.

Theorem 5.1 proves that, for any $k$, there are two values of $p$ for which the linear relationship $D_{k}(p)=\Omega\left(\mathcal{W}_{k}, p\right)-\frac{1}{2}-\frac{1}{2} \Phi\left(\mathcal{W}_{k}, p\right)=0$ holds, and shows the values of $p$ for which this difference $D_{k}(p)$ is positive (or negative). This last point is made explicit in the next corollary. 


\section{Corollary 5.2}

For every $k\left(\frac{n}{2}<k \leq n\right)$ there exists $p_{k} \in\left[\frac{1}{2}, 1\right]$ such that

a) $\Omega\left(\mathcal{W}_{k}, p\right) \geq \frac{1}{2}+\frac{1}{2} \Phi\left(\mathcal{W}_{k}, p\right)$ for any $p \in\left[0, \frac{1}{2}\right] \cup\left[p_{k}, 1\right]$.

b) $\Omega\left(\mathcal{W}_{k}, p\right) \leq \frac{1}{2}+\frac{1}{2} \Phi\left(\mathcal{W}_{k}, p\right)$ for any $p \in\left[\frac{1}{2}, p_{k}\right]$.

As a consequence, it is clear that, except when $n$ is odd and $k=\frac{n+1}{2}$, i.e., when the game is decisive, it is always possible to find two values of $p$ for which the comparison between $\Omega\left(\mathcal{W}_{k}, p\right)$ and $\frac{1}{2}+\frac{1}{2} \Phi\left(\mathcal{W}_{k}, p\right)$ give opposite results. In other words, Corollary 5.2 proves that $D_{k}(p)$ takes positive and negative values for all symmetric games not being decisive, and that decisive symmetric games are the only symmetric games for which $D_{k}(p)$ is always nonnegative, and strictly positive for all $p \neq \frac{1}{2}$. The last corollary in this section points out this fact, i.e., that the only symmetric games in which success exceeds one-half plus one-half times the decisiveness are the decisive symmetric games.

\section{Corollary 5.3}

$\Omega\left(\mathcal{W}_{k}, p\right) \geq \frac{1}{2}+\frac{1}{2} \Phi\left(\mathcal{W}_{k}, p\right)$ for any $p \in[0,1]$ if and only if $\left(N, \mathcal{W}_{k}\right)$ is decisive.

\section{Conclusions}

It is known (see [20], [8]) that the success index $\Omega_{i}(\mathcal{W}, \mathfrak{p})$ and the decisiveness index $\Phi_{i}(\mathcal{W}, \mathfrak{p})$ of a voter $i$ show the linear relationship $\Omega_{i}(\mathcal{W}, \mathfrak{p})=\frac{1}{2}+\frac{1}{2} \Phi_{i}(\mathcal{W}, \mathfrak{p})$, for any voting system $(N, \mathcal{W})$, when the probability $\mathfrak{p}(S)$ of any vote configuration $S$ is uniformly given by $\mathfrak{p}(S)=$ $\frac{1}{2^{n}}$. This relation has somehow contributed to the historical viewing of success as a byproduct of decisiveness. Laruelle and Valenciano proved in [13] that this relationship is not true in general, even for $k$-out-of- $n$ games, which include the majority and the unanimity voting systems. Specifically, they proved that $\Omega_{i}(\mathcal{W}, p)=\frac{1}{2}+\frac{1}{2} \Phi_{i}(\mathcal{W}, p)$ for any simple game $(N, \mathcal{W})$ if and only if $p=\frac{1}{2}$.

In this paper we consider proper $k$-out-of- $n$ games $\left(N, \mathcal{W}_{k}\right)$ and assume that each voter, independently of the others, votes "yes" with probability $p$ and votes "no" with probability $1-p$. In this case the success and the decisiveness indices are the same for any voter $i$ and are respectively denoted by $\Omega\left(\mathcal{W}_{k}, p\right)$ and $\Phi\left(\mathcal{W}_{k}, p\right)$.

In Sections 3 and 4 we consider different proper symmetric games $\left(N, \mathcal{W}_{k}\right)$ and $\left(N, \mathcal{W}_{r}\right)$, with $k<r$, on the same set of voters $N$. Excluding the extreme cases $p=0$ and $p=1$ we prove that the decisiveness in both games coincide for exactly one value of $p, p=p_{k, r} \in$ $\left(\frac{1}{2}, \frac{n-1}{n}\right)$, and that $\Phi\left(\mathcal{W}_{k}, p\right)>\Phi\left(\mathcal{W}_{r}, p\right)$ if and only if $p \in\left(0, p_{k, r}\right)$. Finally it is proved that $\Omega\left(\mathcal{W}_{k}, p\right)>\Omega\left(\mathcal{W}_{r}, p\right)$ for any $p \in(0,1)$, and this shows that there always exist values of $p$ 
for which $\Omega\left(\mathcal{W}_{k}, p\right)>\Omega\left(\mathcal{W}_{r}, p\right)$ and $\Phi\left(\mathcal{W}_{k}, p\right)<\Phi\left(\mathcal{W}_{r}, p\right)$. In particular this happens for any $p \in\left(\frac{n-1}{n}, 1\right)$, independently of the values of $k$ and $r$.

In Section 5 we compare success and decisiveness of a voter in a same game by using $\frac{1}{2}+\frac{1}{2} \Phi\left(\mathcal{W}_{k}, p\right)$ instead of $\Phi\left(\mathcal{W}_{k}, p\right)$. This seems to be an adequate scale to compare these two values since it is known that $\Omega\left(\mathcal{W}_{k}, p\right)=\frac{1}{2}+\frac{1}{2} \Phi\left(\mathcal{W}_{k}, p\right)$ when $p=\frac{1}{2}$. We prove that this relationship holds for two values of $p$, one of them is $p=\frac{1}{2}$, as it was known, and the other one is a value $p_{k} \in\left[\frac{1}{2}, 1\right]$. This value $p_{k}$ reaches its minimum $\frac{1}{2}$ if and only if the game $\left(N, \mathcal{W}_{k}\right)$ is decisive, i.e., $n$ is odd and $k=\frac{n+1}{2}$. We prove that $p_{k}$ increases as $k$ increases and it reaches its maximum value 1 for $k=n$, i.e., for unanimity games. As a consequence we see that $\Omega\left(\mathcal{W}_{k}, p\right) \leq \frac{1}{2}+\frac{1}{2} \Phi\left(\mathcal{W}_{k}, p\right)$ for any $p \in\left[\frac{1}{2}, p_{k}\right]$ and the inequality holds in the opposite sense for the rest of $p \in[0,1]$.

In this paper we have described a simple mathematical procedure to compare the decisiveness and the success of a voter in two different arbitrary symmetric voting games, and to detect when the two notions are in conflict, i.e., they rank voters in an opposite way. Our results can be used to evaluate the respective degrees of success and decisiveness of voters in democratic voting systems, and this would help decision-makers to design appropriate voting systems being able to maximize a good combination between success and decisiveness. It would be also interesting to extend the comparison between success and decisiveness made in this article to any simple game $(N, \mathcal{W})$. In particular, in a future work we will try to identify the games for which these two indices are ordinally equivalent, following the research in [5], [9] and [10].

\section{References}

[1] J.F. Banzhaf. Weighted voting doesn't work: a mathematical analysis. Rutgers Law Review, 19:317-343, 1965.

[2] B. Barry. Is it better to be powerfull or lucky? part i. Political Studies, pages 183-194, 1980.

[3] B. Barry. Is it better to be powerfull or lucky? part ii. Political Studies, pages 338-352, 1980.

[4] S.J. Brams and M. Lake. Power and satisfaction in a representative democracy. In P. Ordeshook, editor, Game Theory and Political Science, pages 529-562. New York University Press, 1978.

[5] F. Carreras and J. Freixas. On ordinal equivalence of power measures given by regular semivalues. Mathematical Social Sciences, 55:221-234, 2008. 
[6] J.S. Coleman. Control of collectivities and the power of a collectivity to act. In B. Lieberman, editor, Social Choice, pages 269-300. Gordon and Breach, New York, USA, 1971.

[7] P. Dubey. On the uniqueness of the Shapley value. American Political Science Review, 4:131-139, 1975.

[8] P. Dubey and L.S. Shapley. Mathematical properties of the Banzhaf power index. Mathematics of Operations Research, 4(2):99-131, 1979.

[9] J. Freixas. On ordinal equivalence of the Shapley and Banzhaf values for cooperative games. International Journal of Game Theory, 39:513-527, 2010.

[10] J. Freixas, D. Marciniak, and M. Pons. On the ordinal equivalence of the Johnston, Banzhaf and Shapley power indices. European Journal of Operational Research, 216:367375, 2012.

[11] G. Gambarelli and I. Stach. Power indices in politics: Some results and open problems. Homo Oeconomicus, 26:417-441, 2009.

[12] T. Köning and T. Bräuninger. The inclusiveness of european decision rules. Canadian Journal of Theoretical Politics, 10:125-142, 1998.

[13] A. Laruelle, R. Martínez, and F. Valenciano. Success versus decisiveness, conceptual discussion and case study. Journal of Theoretical Politics, 18(2):185-205, 2006.

[14] A. Laruelle and F. Valenciano. Assessing success and decisiveness in voting situations. Social Choice and Welfare, 24:171-197, 2005.

[15] A. Laruelle and F. Valenciano. Voting and Collective Decision-Making. Cambridge University Press, 2008.

[16] K. May. A set of independent, necessary and sufficient conditions for simple majority decision. Econometrica, 20:680-684, 1952.

[17] J.W. Mercik. Power and expectations. Control and Cybernetics, 26:617-621, 1997.

[18] G. Owen. Multilinear extensions and the Banzhaf value. Naval Research Logistics Quarterly, 22:741-750, 1975.

[19] M.D. Davis P.D. Straffin and S.J. Brams. Power and satisfaction in an ideologically divided voting body. In M. Holler, editor, Power, voting and voting power, pages 239253. Physica Verlag, WÃrzburg- Wien, 1982. 
[20] L.S. Penrose. The elementary statistics of majority voting. Journal of the Royal Statistical Society, 109:53-57, 1946.

[21] D. Rae. Decision rules and individual values in constitutional choice. American Political Science Review, 63:40-56, 1969.

[22] L.S. Shapley. A value for n-person games. In A.W. Tucker and H.W. Kuhn, editors, Contributions to the theory of games II, pages 307-317. Princeton University Press, Princeton, USA, 1953.

[23] L.S. Shapley and M. Shubik. A method for evaluating the distribution of power in a committee system. American Political Science Review, 48:787-792, 1954.

[24] F. Turnovec. Power, power indices and intuition. Control and Cybernetics, 26:613-615, 1997.

[25] F. Turnovec, J.W. Mercik and M. Mazurkiewicz. Power indices methodology: decisiveness, pivots and swings. In M. Braham and F. Steffen, editors, Power, Freedom and Voting, pages 23-37. Springer Verlag, Berlin, Germany, 2008. 